Whole body computed tomography for trauma patients in the Nordic countries 2014 : survey shows significant differences and a need for common guidelines

Wiklund, E.

2016-06

Wiklund, E , Koskinen, S K , Linder , F , Aslund , P-E \& Eklof , H 2016 , ' Whole body computed tomography for trauma patients in the Nordic countries 2014 : survey shows significant differences and a need for common guidelines ' , Acta Radiologica , vol. 57 , no. 6 , pp. 750-757 . https://doi.org/10.1177/0284185115597718

http://hdl.handle.net/10138/224008

https://doi.org/10.1177/0284185115597718

publishedVersion

Downloaded from Helda, University of Helsinki institutional repository.

This is an electronic reprint of the original article.

This reprint may differ from the original in pagination and typographic detail.

Please cite the original version. 


\title{
Whole body computed tomography for trauma patients in the Nordic countries 20 I 4: survey shows significant differences and a need for common guidelines
}

\author{
E Wiklund', SK Koskinen ${ }^{2,3}$, F Linder ${ }^{4}$, P-E Åslund ${ }^{5}$ and H Eklöf ${ }^{6}$
}

\begin{abstract}
Background: Whole body computed tomography in trauma (WBCTT) is a standardized CT examination of trauma patients. It has a relatively high radiation dose. Therefore, well-defined clinical indications and imaging protocols are needed. This information regarding Nordic countries is limited.

Purpose: To identify Nordic countries' WBCTT imaging protocols, radiation dose, and integration in trauma care, and to inquire about the need for common Nordic guidelines.

Material and Methods: A survey with 23 multiple choice questions or free text responses was sent to 95 hospitals and 10 trauma centers in and outside the Nordic region, respectively. The questions were defined and the hospitals selected in collaboration with board members of "Nordic Forum for Trauma and Emergency Radiology" (www.nordictraumarad.com).

Results: Two Nordic hospitals declined to take part in the survey. Out of the remaining 93 Nordic hospitals, 56 completed the questionnaire. Arterial visualization is routine in major trauma centers but only in $50 \%$ of the Nordic hospitals. The CT scanner is located within $50 \mathrm{~m}$ of the emergency department in all non-Nordic trauma centers but only in $60 \%$ of Nordic hospitals. Radiation dose for WBCTT is in the range of $900-3600 \mathrm{mGy} \times \mathrm{cm}$. Of the 56 responding Nordic hospitals, $84 \%$ have official guidelines for WBCTT. Eighty-nine percent of the responders state there is a need for common guidelines.

Conclusion: Scanning protocols, radiation doses, and routines differ significantly between hospitals and trauma centers. Guideline for WBCTT is presently defined locally in most Nordic hospitals. There is an interest in most Nordic hospitals to endorse new and common guidelines for WBCTT.
\end{abstract}

\section{Keywords}

$\mathrm{CT}$, adults, trauma, radiation safety, equipment, contrast agents - intravenous

Date received: 16 February 2015; accepted: 29 June 2015

\section{Introduction}

Standardized computed tomography (CT) protocol of head-thorax-abdomen was introduced to Nordic countries in the late 1990s (1), and is now referred to as whole body CT in trauma (WBCTT). Standardized CT protocols have significantly shortened time in the emergency room and the time that patients spend in emergency departments (2-8). Therefore, WBCTT has gained popularity and is currently being performed routinely in many hospitals receiving trauma patients $(9,10)$. However, WBCTT
'Faculty of Medicine, Uppsala University, Uppsala, Sweden

${ }^{2}$ Department of Radiology, Helsinki University Central Hospital, Helsinki Medical Imaging Center, Helsinki, Finland

${ }^{3}$ Division of Medical Imaging and Technology, Department of Clinical Science, Intervention and Technology, Department of Radiology,

Karolinska Institutet and Karolinska University Hospital Huddinge

${ }^{4}$ Department of Surgical Sciences, Section of Vascular Surgery, Uppsala University, Uppsala, Sweden

${ }^{5}$ Department of Medical Physics, Uppsala University Hospital, Uppsala University, Uppsala, Sweden

${ }^{6}$ Department of Surgical Sciences, Section of Radiology, Uppsala

University, Uppsala, Sweden

Corresponding author:

Hampus Eklöf, Department of Radiology, Uppsala University hospital, SE75I 85 Uppsala, Sweden.

Email: hampus.eklof@radiol.uu.se 
exposes the patient to a relatively high radiation dose, and the number of patients exposed to a radiation dose of 20 millisieverts (mSv) has increased (11), and there have been concerns over the increased use of CT (12).

A variety of criteria are used as indications for performing WBCTT, e.g. mechanism of injury, abnormal vital signs, and/or clinically apparent injuries, and different scanning protocols can be used as well (12-16). Therefore, to optimize trauma care, local clinical decision rules or guidelines should be applied. However, a survey conducted 2011 in the UK to identify the use of WBCTT showed that few hospitals in the UK had official guidelines $(22 \%)$, but a similar proportion $(23 \%)$ used WBCTT without the presence of official guidelines (17). To our knowledge it is not generally known how WBCTT is currently used in the Nordic countries. Therefore, the aim of this study was to find out how WBCTT is used in the Nordic countries today and is there a need for common guidelines?

\section{Material and Methods}

A survey was sent to 95 Nordic hospitals. The radiologist in charge of emergency radiology or CT was asked to complete the questionnaire. The selection of hospitals was based on web-search, and hospitals with emergencies departments where name and address to trauma radiologist or head of radiology department could be found: Sweden $(n=54)$, Denmark $(n=21)$, Norway $(n=5)$, and Iceland $(n=2)$. In Finland, 13 major hospitals in trauma care were selected by a "Nordic Forum for Trauma and Emergency Radiology (NORDTER)" (www.nordictraumarad.com/) board member with personal knowledge of whom to contact The survey was also sent to 10 major trauma centers in the USA $(n=5)$, the Netherlands $(n=2)$, Belgium $(n=1)$, Italy $(n=1)$, and England $(n=1)$ that were known to be active in trauma imaging research. NORDTER board members selected the non-Nordic trauma-centers and assisted in selecting the Nordic hospitals. The questionnaire comprised 23 multiple choice and free text response questions (see Appendix 1 - online only). The questionnaire was put together using Google forms online to enable easy distribution and collection of answers. Participants were given the option of getting the questionnaire sent by regular mail and reminders were sent two times to hospitals that did not reply to ensure maximized answering frequency.

Statistical analysis was made using basic spreadsheet functions of Microsoft Excel 2013 (Microsoft Corp, Redmond, WA, USA).

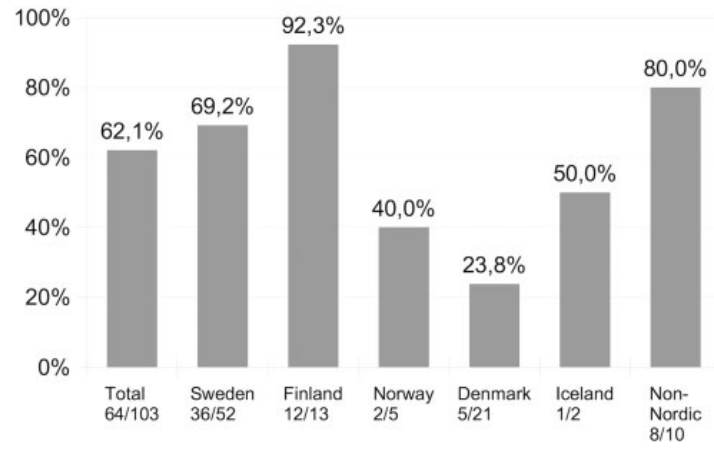

Fig. I. Answering frequency in total and for each country/ region.

\section{Results}

\section{Response rate}

Two hospitals in Sweden responded that trauma patients are taken to other hospitals in the region and therefore declined to take part in the survey. Of the remaining 93 Nordic hospitals, $56(60.2 \%)$ completed the questionnaire. The frequency of answers for each country/region is presented in Fig. 1.

\section{Number of WBCTTs performed and official guidelines}

The hospitals were asked to approximate the number of WBCTT they perform on average (Table 1).

An alert policy for severely ill trauma patients that can be set off either from the emergency department or the local ambulance service to optimize the staffing in the emergency room was present in $52(96.4 \%)$ of the 56 responding Nordic hospitals and in seven $(87.5 \%)$ of the eight non-Nordic hospitals. An official guideline for when it is indicated to perform a WBCTT was present in $47(83.9 \%)$ of the Nordic hospitals (Table 2$)$ and in six $(75.0 \%)$ of the non-Nordic hospitals. Three of the hospitals (located in the USA, Norway, and Iceland) that do not have a guideline are in the process of defining guidelines.

Of all the hospitals that responded, 46 (71.9\%) perform WBCTT after the primary survey. Data for when WBCTT is performed in Nordic and non-Nordic countries are presented in Fig. 2.

\section{Criteria for performing WBCTT}

The criteria used for deciding if WBCTT should be performed varied between hospitals (Table 3 ). The most common criterion used was mechanism of injury. Three hospitals responded that they only use this specific criterion. 
Table I. Number of WBCTTs performed and number of Nordic hospitals that have official guidelines.

\begin{tabular}{lccc}
\hline WBCTTs performed $(n)$ & Nordic hospitals, $n(\%)$ & $\begin{array}{l}\text { Non-Nordic } \\
\text { hospitals, } n(\%)\end{array}$ & $\begin{array}{l}\text { Nordic hospitals with } \\
\text { official guidelines, } n(\%)\end{array}$ \\
\hline Fewer than I per week & $5(8.8 \%)$ & 0 & $4(80.0 \%)$ \\
I-3 per week & $16(28.6 \%)$ & $1(12.5 \%)$ & $15(93.4 \%)$ \\
4-6 per week & $16(28.6 \%)$ & 0 & $14(87.5 \%)$ \\
About I per day & $7(12.5 \%)$ & 0 & $4(57.1 \%)$ \\
I-3 per day & $10(17.9 \%)$ & $3(37.5 \%)$ & $8(80.0 \%)$ \\
4-6 per day & $2(3.6 \%)$ & $3(37.5 \%)$ & $2(100.0 \%)$ \\
More than 6 per day & 0 & $1(12.5 \%)$ & $n / a$ \\
\hline
\end{tabular}

Table 2. Guidelines for indications to perform WBCTT and distribution for different hospital categories in the Nordic countries.

\begin{tabular}{lllll}
\hline Official guidelines for WBCTT & Hospitals, $n(\%)$ & University hospital & District hospital & Local hospital \\
\hline Departmental guidelines & $27(48.2 \%)$ & $9 / 16$ & $13 / 29$ & $5 / 11$ \\
Regional guidelines & $16(28.6 \%)$ & $1 / 16$ & $12 / 29$ & $3 / 11$ \\
Both & $4(7.1 \%)$ & $2 / 16$ & $2 / 29$ & 0 \\
No & 0 & 0 & 0 & 0 \\
No guidelines, but use WBCTT & $8(14.3 \%)$ & $4 / 16$ & $2 / 29$ & $2 / 11$ \\
Don't know & I (I.8\%) & 0 & 0 & $1 / 11$ \\
\hline
\end{tabular}

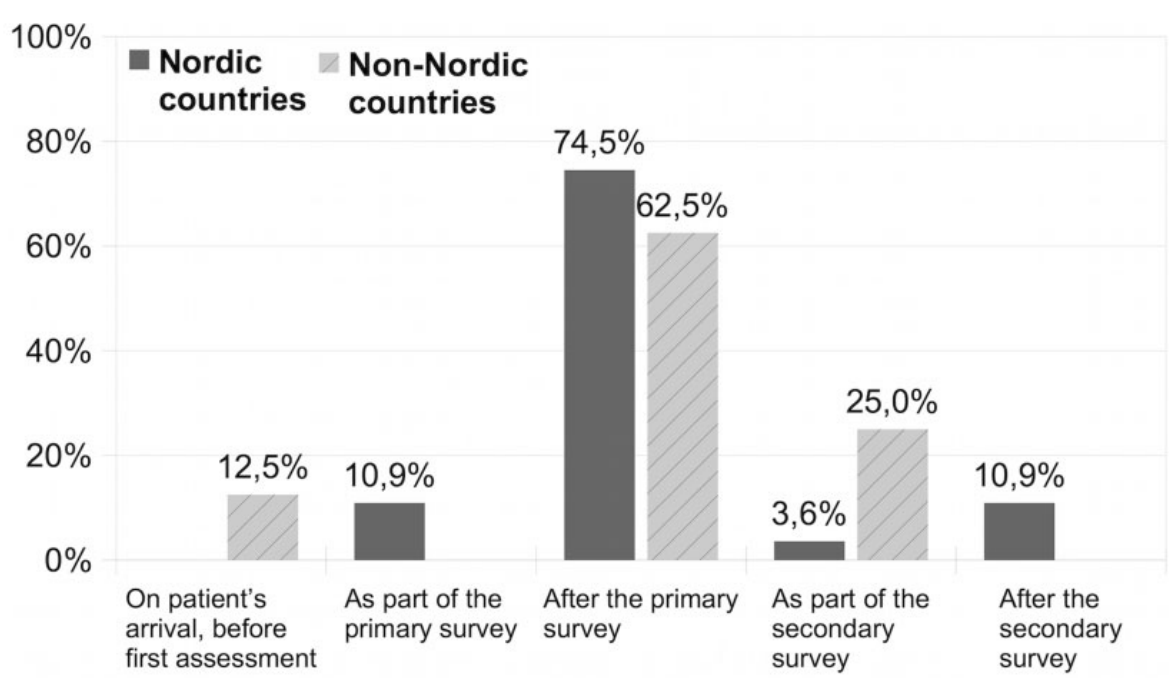

Fig. 2. When WBCTT is performed.

\section{Reporting of WBCTT}

The survey showed that reporting in Nordic countries is done both by consultants and residents (Fig. 3). If a resident makes the initial radiology report, $50(89.3 \%)$ of the Nordic hospitals reported that a consultant makes the final report or comments it.

Three hospitals in Sweden commented that outof-hours the reporting is done by radiologists at a teleradiology center in Sydney, Australia, including
WBCTT unless if it is associated with a trauma alarm. In that case, a local radiologist who is on standby at home will come to the hospital.

The non-Nordic trauma centers responded that a consultant does in-hours reporting in three (37.5\%) hospitals and by either a consultant or a resident in the remaining five $(62.5 \%)$ hospitals. During out-of-hours, in six $(75.0 \%)$ hospitals the reports are made by a resident and by consultants at two $(25.0 \%)$ hospitals. If a resident made the initial report, then a consultant makes 
the final report in seven $(87.5 \%)$ hospitals. A majority of the Nordic hospitals responded that they report results of WBCTT orally and make provisional and final reports to the doctor in charge (Fig. 4).

Table 3. Criteria used for deciding if WBCTT should be performed.

\begin{tabular}{llll}
\hline & $\begin{array}{l}\text { Nordic } \\
\text { hospitals, } \\
n(\%) *\end{array}$ & $\begin{array}{l}\text { Non-Nordic } \\
\text { hospitals, } \\
n(\%) *\end{array}$ & Total (\%)* \\
\hline $\begin{array}{c}\text { Criterion } \\
\begin{array}{c}\text { Mechanism } \\
\text { of injury }\end{array}\end{array}$ & $52(94.5 \%)$ & $6(75.0 \%)$ & $58(92.1 \%)$ \\
$\begin{array}{c}\text { Abnormal } \\
\text { vital signs }\end{array}$ & $49(89.1 \%)$ & $7(87.5 \%)$ & $56(88.9 \%)$ \\
$\begin{array}{c}\text { Anatomical } \\
\text { site of injury }\end{array}$ & $37(67.3 \%)$ & $5(62.5 \%)$ & $42(66.7 \%)$ \\
$\begin{array}{c}\text { Presence of } \\
\text { multiple injuries }\end{array}$ & $46(83.6 \%)$ & $7(87.5 \%)$ & $53(84.1 \%)$ \\
\begin{tabular}{c} 
Other \\
\hline
\end{tabular} & $7(12.7 \%)$ & $3(37.5 \%)$ & $10(15.9 \%)$ \\
\hline
\end{tabular}

*Multiple choices allowed.

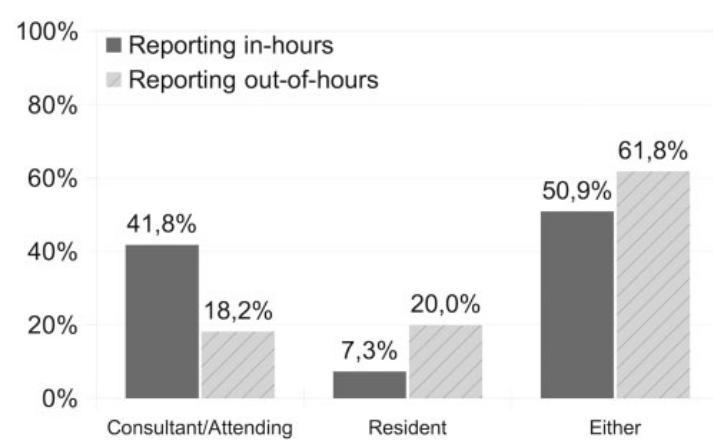

Fig. 3. Who reports WBCTT scans in- and out-of-hours in Nordic hospitals.

\section{Protocols for WBCTT}

All hospitals answered that they use intravenous contrast routinely. A standardized volume of i.v. contrast was used in $7 / 8(87.5 \%)$ of the non-Nordic trauma centers. In the Nordic hospitals 30 of $55(54.5 \%)$ use a standardized dose and $25(45.5 \%)$ personalize the dose depending on age, weight, gender, and renal function. All were asked to describe how the different body parts are visualized with respect to i.v. contrast (Tables 4 and 5). For upper and lower abdomen excretoryphase was given as an option and for lower extremity the option "this body part is not included routinely".

\section{Location of CT scanner}

The CT scanner located inside or adjacent to the emergency department (ED) was present in $33(60.0 \%)$ of the responding Nordic hospitals. Adjacent was defined as on the same floor and within $50 \mathrm{~m}$ from the ED. All non-Nordic trauma centers had a CT scanner located near the ED, two commented that the scanner is located inside the trauma room.

\section{Alignment of arms}

The hospitals were asked to submit how the patient's arms are aligned when performing WBCTT. Results for the Nordic hospitals are presented in Fig. 5.

\section{Radiation}

The average radiation dose to the patient during WBCTT defined as the average DLP (milligray [mGy] $\times \mathrm{cm})$ was reported by $37(60 \%)$ of responding hospitals and four $(50 \%)$ of the responding trauma centers. This is presented in Table 6 .

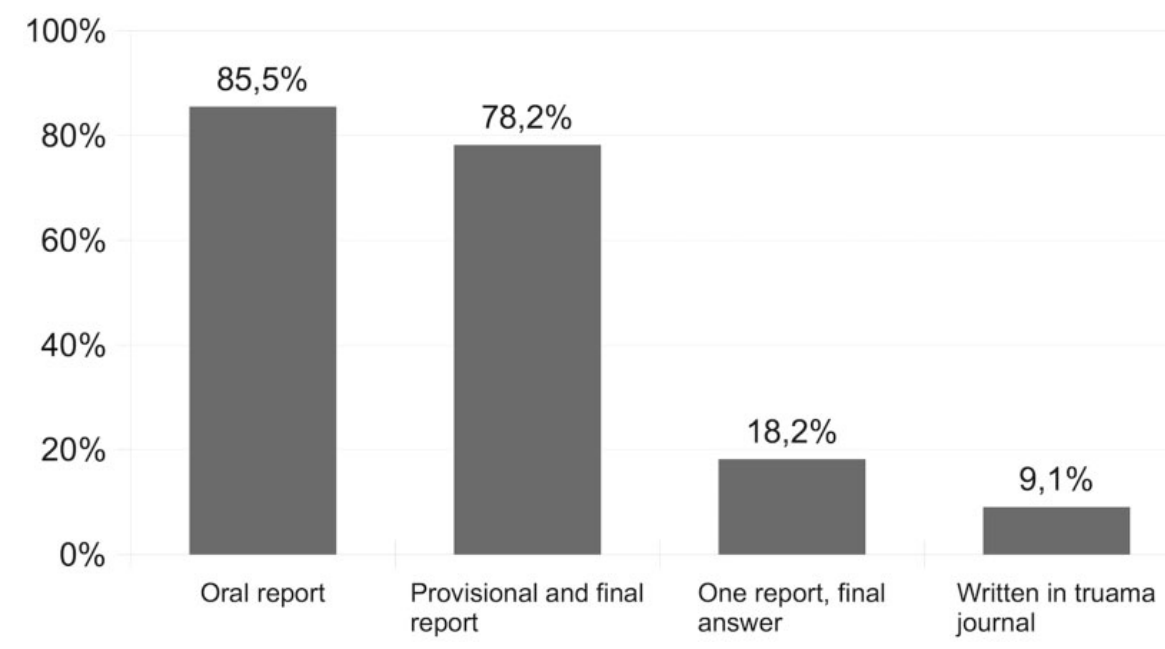

Fig. 4. How results of WBCTT are reported in Nordic hospitals. Multiple choices allowed. 
Table 4. How different body parts are visualized in Nordic hospitals (multiple choices allowed).

\begin{tabular}{|c|c|c|c|c|c|}
\hline Body part & $\begin{array}{l}\text { Without } \\
\text { contrast }\end{array}$ & $\begin{array}{l}\text { Arterial } \\
\text { phase }\end{array}$ & Venous phase & $\begin{array}{l}\text { Combined arterial } \\
\text { and venous phase }\end{array}$ & Excretory phase \\
\hline Head & 54 (98.2\%) & I (I.8\%) & 0 & 0 & $\mathrm{n} / \mathrm{a}$ \\
\hline Neck & $42(76.4 \%) *$ & $7(12.7 \%)$ & $3(5.5 \%)$ & $3(5.5 \%)^{\dagger}$ & $\mathrm{n} / \mathrm{a}$ \\
\hline Thorax & 0 & $19(34.5 \%)$ & $26(47.3 \%)$ & $10(18.2 \%)$ & $\mathrm{n} / \mathrm{a}$ \\
\hline Upper abdomen & 0 & $9(16.4 \%)$ & $28(50.9 \%)$ & $15(27.3 \%)$ & $9(16.4 \%)^{\S}$ \\
\hline Lower abdomen (including pelvis) & 0 & $6(10.9 \%)$ & $33(60.0 \%)$ & $12(21.8 \%)$ & $9(16.9 \%)^{\S}$ \\
\hline Lower extremity** & 0 & 0 & $\mathrm{I}(\mathrm{I} .8 \%)$ & $\mathrm{I}(\mathrm{I} .8 \%)$ & $\mathrm{n} / \mathrm{a}$ \\
\hline
\end{tabular}

*Eight (14.5\%) of the hospitals added that they perform neck scanning in arterial phase (CT angiography) if there is any sign of cervical injury in the first scan.

${ }^{\top}$ Two of the hospitals use a split bolus technique.

Six of the hospitals that combine arterial and venous phase commented that they use a split bolus technique.

${ }^{\S}$ Seven of the hospitals that scan in excretory phase do this if there is a suspected renal collecting system injury.

**Fifty-three (96.4\%) do not include lower extremities routinely, but perform scans if there are suspected injuries.

Table 5. How different body parts are visualized in non-Nordic trauma centers (multiple choices allowed).

\begin{tabular}{|c|c|c|c|c|c|}
\hline Body part & Without contrast & $\begin{array}{l}\text { Arterial } \\
\text { phase }\end{array}$ & Venous phase & $\begin{array}{l}\text { Combined arterial } \\
\text { and venous phase }\end{array}$ & $\begin{array}{l}\text { Excretory } \\
\text { phase }\end{array}$ \\
\hline Head & $7(87.5 \%)$ & 0 & 0 & I (I2.5\%)* & $\mathrm{n} / \mathrm{a}$ \\
\hline Neck & $\mathrm{I}(\mathrm{I} 2.5 \%)$ & $5(62.5 \%)$ & 0 & $2(25.0 \%)^{*}$ & $\mathrm{n} / \mathrm{a}$ \\
\hline Thorax & 0 & $5(62.5 \%)$ & 0 & $3(37.5 \%) *$ & $\mathrm{n} / \mathrm{a}$ \\
\hline Upper abdomen & 0 & $\mathrm{I}(12.5 \%)$ & 0 & $7(87.5 \%)^{\dagger}$ & 0 \\
\hline Lower abdomen (incl. pelvis) & 0 & $3(37.5 \%)$ & $\mathrm{I}(12.5 \%)$ & $4(50.0 \%)^{\dagger}$ & 0 \\
\hline Lower extremity & 0 & $3(37.5 \%)$ & 0 & I $(12.5 \%)^{*}$ & $\mathrm{n} / \mathrm{a}$ \\
\hline
\end{tabular}

*Performed with split bolus technique.

Three of the hospitals use split bolus.

Four of the hospitals do not include lower extremities routinely, but perform scans if there are suspected injuries.

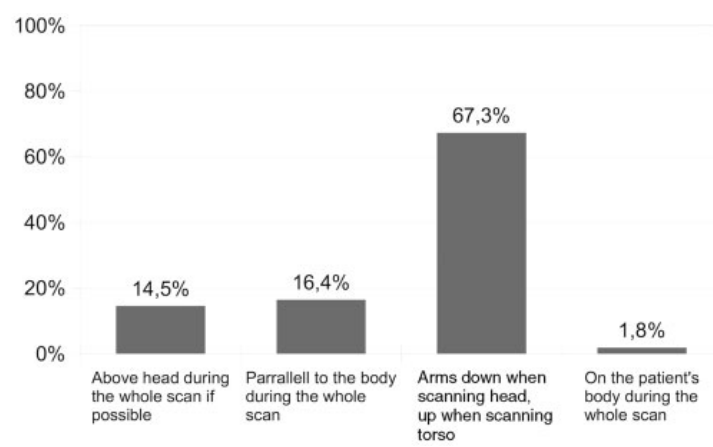

Fig. 5. How the patient's arms are aligned during WBCTT in Nordic hospitals.

\section{Need for national/international guidelines}

To conclude the survey the responders were asked to answer if they think there is a need for national/international guidelines concerning WBCTT. They were also asked if their department would be interested in adopting new guidelines in the future. Data are displayed in Fig. 6.
Table 6. Radiation dose (DLP; $m G y \times c m)$ from WBCTT in Nordic hospitals and in non-Nordic trauma centers.

\begin{tabular}{lccccc}
\hline & $\begin{array}{l}\text { Hospitals } \\
(n)\end{array}$ & Maximum & Minimum & Mean & SD \\
\hline Nordic & 33 & 3600 & 900 & 1838 & 535 \\
Non-Nordic & 4 & 2750 & 1700 & 2200 & 505 \\
\hline
\end{tabular}

\section{Discussion}

This survey had a response rate of $62.1 \%$. This is a normal response rate frequently reported when questionnaires are sent to physicians (18). Answering frequency was low for Denmark and Norway and thus it can be questioned if their answers are representative. Also, the web-search found more Swedish hospitals than, for example, Norwegian ones, and this may be a source of bias. The survey received good response rates in Sweden, Finland, and the non-Nordic region and it is less likely that non-response bias is a problem in these countries. No follow-up has been done to 


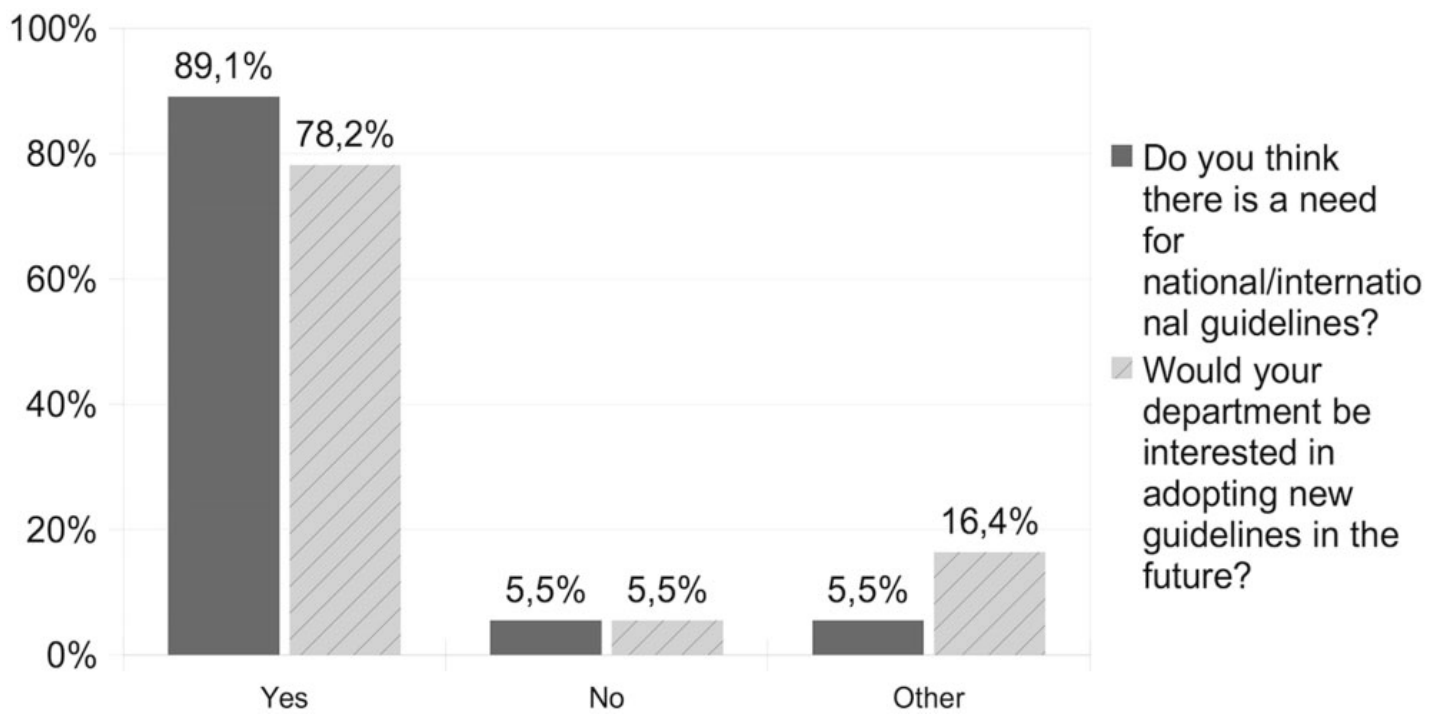

Fig. 6. Need for national/international guidelines and interest in adopting new guidelines in Nordic hospitals.

examine how the non-responders differ in routines. It is possible that the response rate would have increased if more efforts were made, for example making phone calls to the hospitals that did not respond. This survey displays the present situation in the Nordic hospitals, but does not provide any answers to the appropriateness of the routines currently used.

Of the 56 responding Nordic hospitals, 47 (83.9\%) have official guidelines for when the use of WBCTT is indicated. This was higher than expected as a similar study found guidelines for WBCTT indications in only $22.3 \%$ in the UK (17). Even though most of the Nordic hospitals have a current guideline, $49(89.1 \%)$ of the responders believe that there is a need for national or international guidelines and a majority are also interested in adopting new national or international guidelines in the future. The present survey indicates major differences in protocols and radiation doses for WBCTT between Nordic hospitals and even more when compared with the non-Nordic trauma centers. However, this study did not take into account the type of the CT scanner (i.e. 16- or 64-slice, etc.) which may affect scanning protocol and radiation dose the protocol selection and radiation dose. Also, the selection of non-Nordic sites was not randomized, and this might be a source of selection bias.

Fifty-three $(82.8 \%)$ of the responding hospitals have official guidelines for the use of WBCTT and 10 $(15.9 \%)$ of the hospitals without official guidelines perform WBCTT if it is considered adequate by the doctor in charge of the trauma. Forty-seven $(83.9 \%)$ of the Nordic hospitals have official guidelines and six $(75.0 \%)$ of the non-Nordic hospitals. In a similar study made in the UK (17) $22.3 \%$ of the hospitals had official guidelines and another $23.4 \%$ used
WBCTT without guideline. This study was conducted in 2011 and therefore do not necessarily represent the current situation in the UK. The current survey shows that the hospitals in the Nordic region have come a far way in composing guidelines. Even so there seem to be little consensus between hospitals for how these guidelines should be formulated.

Most Nordic hospitals scan the patient only once while major trauma centers recommend repeated scanning of selected parts of the body (scanning head first before i.v. contrast, then scanning in arterial phase of head-neck-thorax-abdomen, and an additional scan of the abdomen in venous phase). Scanning a late phase is optional when injury of renal collecting system is expected. Split-bolus technique is questioned as it might hide pseudoaneurysms of the spleen and liver (19).

This study demonstrates major differences in WBCTT protocols used in Nordic hospitals. There were also differences between the Nordic and the nonNordic hospitals included in this survey. An important difference was the scanning of the neck with contrast enhancement of arteries. It has been shown that the use of contrast when scanning the neck and cervical spine is useful for detecting arterial injuries and it is recommended if there are documented injuries of the head and neck (20). This was done regularly in only $18 \%$ of the Nordic hospitals compared to $88 \%$ of the nonNordic hospitals. Another eight (14.5\%) Nordic hospitals scan the neck in arterial phase if there are signs of injuries. As seen in Table 5 almost all of the nonNordic hospitals perform WBCTT with visualization of arteries from neck to pelvis. Few Nordic hospitals reported a similar protocol. One possible reason for the difference between the Nordic and the non-Nordic hospital is that the non-Nordic hospitals are exposed to 
a different spectrum of trauma, e.g. penetrating gunshot injuries are more common in the non-Nordic countries.

Biphasic injection of contrast media resulting in contrast enhancement of arteries, veins, and organs was used in six $(10.9 \%)$ of the Nordic hospitals. Monophasic injection of contrast media with scanning twice was performed in nine of the Nordic hospitals. Radiation dose is reduced when biphasic or a triphasic injection are used as this reduces the number of scans. However, these techniques are far from optimal in detecting splenic and hepatic pseudoaneurysms and arteriovenous (AV) fistula (19).

Hospitals are using multiple criteria for deciding when to perform WBCTT $(13,21)$. This also applies to the Nordic region. Most of the Nordic hospitals use mechanism of injury as a criterion for deciding when to perform WBCTT. The criteria reported in the current study were also reported in a survey made in the UK (17). Their most reported criterion was presence of multiple injuries $(90.2 \%)$ followed by mechanism of injury (75.6\%), abnormal vital signs $(65.9 \%)$, and anatomical site of injury (56.1\%). Compared with the Nordic hospitals, presence of multiple injuries is a more frequently used criterion in the UK. The three other criteria (mechanism of injury, abnormal vital signs, and anatomical site of injury) are more frequently used in the Nordic countries. Compared with the nonNordic hospitals, the Nordic hospitals reported that mechanism of injury is a more frequently used criterion. The other criteria were used in similar proportions of the hospitals. It is difficult to determine which criterion is more adequate since there seems to be no distinct consensus in the literature. As shown earlier, the criteria reported in this survey have a high sensitivity, but a low specificity and may lead to unnecessary scans (13).

A CT scanner was located inside or adjacent to the ED in a majority $(60.0 \%)$ of the Nordic hospitals, which is almost identical to a UK study (17). All of the non-Nordic major trauma centers included in this current survey had a CT scanner located inside or near the ED. Of the Nordic hospitals, $10.9 \%$ are currently planning to relocate a CT scanner closer to the ED. We expect more hospitals will consider having a CT scanner close to the ED. The time before scan was reduced by $25 \%$ and the number of transports of the patients was reduced by more than half if the CT scanner was located in the trauma room (21).

The radiation dose of WBCTT to the patient can be estimated using the dose length product (DLP) recorded for each scan. In the Nordic hospitals DLP was in the range of 900-3600 with an average of $1838 \pm 535 \mathrm{mGy} \times \mathrm{cm}$. The DLP was higher in the non-Nordic hospitals with an average of $2200 \pm 505 \mathrm{mGy} \times \mathrm{cm}$. Three of the Nordic hospitals and one of the non-Nordic hospitals provided a DLP that was higher than $2500 \mathrm{mGy} \times \mathrm{cm}$. The effective radiation dose to the patient, however, was not evaluated in this study as it depends on several factors that were not taken into account. Radiation dose is related to the number of scans of each body part, dose/scan, dose reduction features of the specific scanner, and positioning of arms during scanning. Realigning the patient's arms when scanning the thorax and abdomen could reduce the effective dose by $16-22 \%(22,23)$. Also, if arms are parallel to the body, it has a negative effect on image quality (24). In our study, $16 \%$ of the Nordic hospitals reported that they perform WBCTT with the patient's arms parallel to the body during the whole scan. The presence of spine-board under the patient and metal frames stabilizing fractures also induces higher radiation doses.

The effective radiation dose to the patient is a measure taking into account the sensitivity to radiation of different tissues. Assuming that the DLP to the head was approximately $1200 \mathrm{mGy} \times \mathrm{cm}$ (the average DLP for head from our hospital for WBCTT) and the remaining DLP belongs to thorax/abdomen, the effective dose was estimated to $14 \mathrm{mSv}, 21 \mathrm{mSv}$, and $26 \mathrm{mSv}$ for a DLP of $1838 \mathrm{mGycm}, 2200 \mathrm{mGycm}$, and $2500 \mathrm{mGycm}$, respectively. For the estimation of effective dose of thorax and abdomen, the conversion factor was chosen to 0.018 (average of 0.017 and 0.019) $(25,26)$. This will probably overestimate the effective dose since the scan over the neck will be calculated as thorax/abdomen.

It remains a challenge to find the correct indication for when WBCTT should be performed. It is still to be shown definitively if WBCT for trauma patients has an effect on morbidity and mortality. The REACT-2 study (15) might provide some answers to this. The current survey indicates a will to accept and a need for Nordic guidelines regarding WBCTT. However, many considerations must be taken into account to make the guidelines adoptable for hospitals with different CT scanners. Hopefully, guidelines will contribute to better trauma care, shorter time to correct diagnosis, optimized scanning protocols, and reduced radiation exposure. Due to potentially high radiation dose from WBCTT to the usually young trauma patients and the introduction of new scanning routines from major trauma centers, guidelines should be encouraged. Guidelines for WBCTT would ensure updated protocols in all hospitals and facilitate consultation between hospitals, which should benefit the patients of trauma.

In conclusion, WBCTT is a scanning protocol for quick and expeditious CT examination of trauma patients, presently defined locally in most Nordic trauma hospitals. Scanning protocols differ significantly between centers. There is an interest in most Nordic hospitals to endorse new guidelines for WBCTT. 


\section{Acknowledgements}

We would like to express our appreciation to Dr Christopher M Smith, author of a similar survey made in the UK. He provided us with the questionnaire they used and helpful advice for this study.

\section{Conflict of interest}

None declared.

\section{Funding}

This research received no specific grant from any funding agency in the public, commercial, or not-for-profit sectors.

\section{References}

1. Leidner B, Beckman MO. Standardized whole-body computed tomography as a screening tool in blunt multitrauma patients. Emerg Radiol 2001;8:20-28.

2. Sierink JC, Saltzherr TP, Reitsma JB, et al. Systematic review and meta-analysis of immediate total-body computed tomography compared with selective radiological imaging of injured patients. Br J Surg 2012;99(Suppl. 1): $52-58$.

3. Wurmb TE, Frühwald P, Hopfner W, et al. Whole-body multislice computed tomography as the first line diagnostic tool in patients with multiple injuries: the focus on time. J Trauma 2009;66:658-665.

4. Rieger M, Czermak B, El Attal R, et al. Initial clinical experience with a 64-MDCT whole-body scanner in an emergency department: better time management and diagnostic quality? J Trauma 2009;66:648-657.

5. Trupka A, Waydhas C, Hallfeldt KK, et al. Value of thoracic computed tomography in the first assessment of severely injured patients with blunt chest trauma: results of a prospective study. J Trauma 1997;43:405-11. (discussion 411-412).

6. Brown CVR, Antevil JL, Sise MJ, et al. Spiral computed tomography for the diagnosis of cervical, thoracic, and lumbar spine fractures: its time has come. J Trauma 2005;58:890-95. (discussion 895-896).

7. Pinto A, Niola R, Tortora G, et al. Role of multidetectorrow $\mathrm{CT}$ in assessing the source of arterial haemorrhage in patients with pelvic vascular trauma. Comparison with angiography. Radiol Med 2010;115:648-667.

8. Holmes JF, Akkinepalli R. Computed tomography versus plain radiography to screen for cervical spine injury: a meta-analysis. J Trauma 2005;58:902-905.

9. Sierink JC, Saltzherr TP, Wirtz MR, et al. Radiation exposure before and after the introduction of a dedicated total-body CT protocol in multitrauma patients. Emerg Radiol 2013;20:507-512.

10. Linsenmaier U, Geyer LL, M, Körner M, et al. Importance of multidetector CT imaging in multiple trauma. Radiologe 2014;54:861-871.

11. Asha S, Curtis KA, Grant N, et al. Comparison of radiation exposure of trauma patients from diagnostic radiology procedures before and after the introduction of a panscan protocol. Emerg Med Australas 2012;24:43-51.
12. Increased use of CT causes concern in the Nordic countries. Available at: http://www.stuk.fi/stuk/tiedotteet/ 2012/en_GB/news_709/(accessed 17 February 2014)

13. Wurmb TE, Frühwald P, Hopfner W, et al. Whole-body multislice computed tomography as the primary and sole diagnostic tool in patients with blunt trauma: searching for its appropriate indication. Am J Emerg Med 2007;25: 1057-1062.

14. Deunk J, Brink M, Dekker HM, et al. Routine versus selective computed tomography of the abdomen, pelvis, and lumbar spine in blunt trauma: a prospective evaluation. J Trauma 2009;66:1108-1117.

15. Huber-Wagner S, Biberthaler P, Häberle S, et al. Wholebody CT in haemodynamically unstable severely injured patients-a retrospective, multicentre study. PloS One 2013;8:e68880.

16. Sierink JC, Saltzherr TP, Beenen LF, et al. A multicenter, randomized controlled trial of immediate total-body CT scanning in trauma patients (REACT-2). BMC Emerg Med 2012;12:4.

17. Smith CM, Mason S. The use of whole-body CT for trauma patients: survey of UK emergency departments. Emerg Med 2012;29:630-634.

18. Cummings SM, Savitz LA, Konrad TR. Reported response rates to mailed physician questionnaires. Health Serv Res 2001;35:1347-1355.

19. Boscak AR, Shanmuganathan K, Mirvis SE, et al. Optimizing trauma multidetector CT protocol for blunt splenic injury: need for arterial and portal venous phase scans. Radiology 2013;268:79-88.

20. Fleck SK, Langner S, Baldauf J, et al. Incidence of blunt craniocervical artery injuries: use of whole-body computed tomography trauma imaging with adapted computed tomography angiography. Neurosurgery 2011;69: 615-23. (discussion 623-624).

21. Saltzherr TP, Bakker FC, Beenen LF, et al. Randomized clinical trial comparing the effect of computed tomography in the trauma room versus the radiology department on injury outcomes. Br J Surg 2012;99(Suppl. 1): 105-113.

22. Loewenhardt B, Buhl M, Gries A, et al. Radiation exposure in whole-body computed tomography of multiple trauma patients: bearing devices and patient positioning. Injury 2012;43:67-72.

23. Brink M, de Lange F, Oostveen LJ, et al. Arm raising at exposure-controlled multidetector trauma CT of thoracoabdominal region: higher image quality, lower radiation dose. Radiology 2008;249:661-670.

24. Karlo C, Gnannt R, Frauenfelder T, et al. Whole-body $\mathrm{CT}$ in polytrauma patients: effect of arm positioning on thoracic and abdominal image quality. Emerg Radiol 2011;18:285-293.

25. Huda W, Ogden KM, Khorasani MR. Converting doselength product to effective dose at CT. Radiology 2008; 248:995-1003.

26. Deak PD, Smal Y, Kalender WA. Multisection CT protocols. Sex and age specific conversion factors used to determine effective dose from dose-length product. Radiology 2010;257:158-166. 